\title{
Image 2:039 $\quad$ Reducing Corneal Astigmatism Using Cataract and LRI Incisions
}

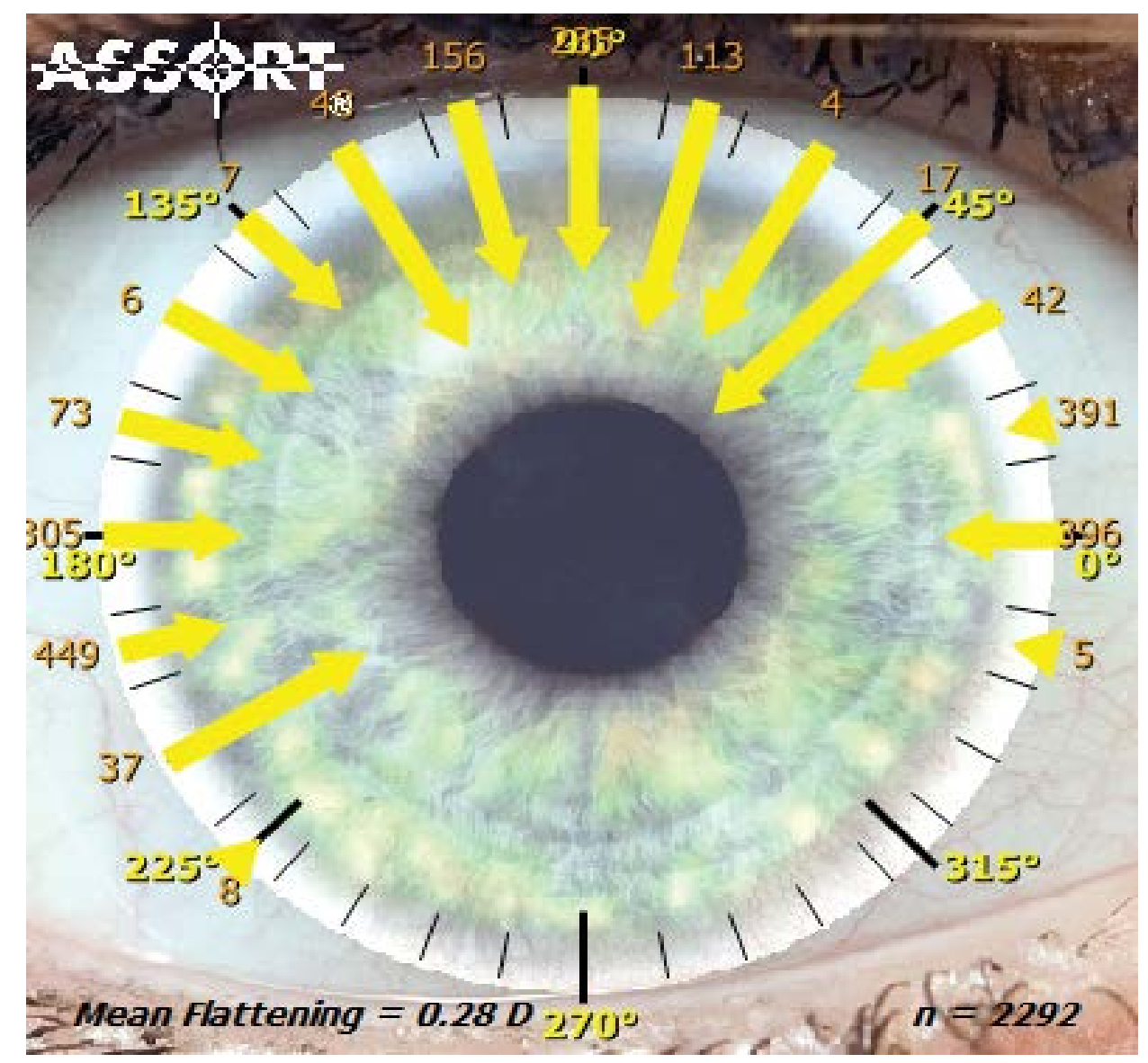

Figure 1: Analysis of flattening effect (FE) of primary incisions for cataract surgery in both right and left eyes. The flattening effect can be used for future toric IOL and femto-LRI planning procedures.

Astigmatism analysis of corneal incisions can be used to reduce the amount of corneal astigmatism at the time of surgery. By comparing postoperative versus preoperative corneal astigmatism vectorially, the flattening effect (FE) can be calculated and be used as a guide for future surgery. Placing the incision on the steepest corneal meridian will maximally reduce the astigmatism, while placing it away from the steepest corneal meridian will rotate the axis of the astigmatism and reduce the magnitude to a lesser amount.

The image displays the flattening effect at 10 degree intervals of incisions for right and left eyes using a $2.2 \mathrm{~mm}$ clear corneal incision. The numbers around the limbus indicate how many cases were performed at any particular 10 degree position; the longer the arrow, the more the flattening effect. It is interesting to note that there is more flattening effect vertically (at 90 degrees) than horizontally (0-180 degrees) (Figure 1).

\section{Information}

\section{Noel Alpins*}

Department of Ophthalmology, Melbourne University, Australia

*Correspondence: Noel Alpins, Medical Director, NewVision Clinics, Clinical Senior Lecturer, Department of Ophthalmology, Melbourne University, 7 Chesterville Road, Cheltenham, Victoria 3192, Australia, Tel: 613 9584 6122, E-mail: alpins@newvisionclinics.com.au
Citation: Alpins N (2016) Reducing Corneal Astigmatism Using Cataract and LRI Incisions. Clin Med Img Lib 2:039

Published: April 22, 2016

Copyright: ( 2016 Alpins N. This is an open-access content distributed under the terms of the Creative Commons Attribution License, which permits unrestricted use, distribution, and reproduction in any medium, provided the original author and source are credited. 